\title{
UCRL-SR-236551
}

LAW RENCE LIVERMORE N A T IO N A L LABORATORY

\section{Comparison of observed and theoretical Fe $L$ emission from CIE plasmas}

M. Carpenter, P. Beiersdorfer, G. V. Brown, H. C. Chen, M. F. Gu, J. G. Jernigan

November 14, 2007 
This document was prepared as an account of work sponsored by an agency of the United States government. Neither the United States government nor Lawrence Livermore National Security, LLC, nor any of their employees makes any warranty, expressed or implied, or assumes any legal liability or responsibility for the accuracy, completeness, or usefulness of any information, apparatus, product, or process disclosed, or represents that its use would not infringe privately owned rights. Reference herein to any specific commercial product, process, or service by trade name, trademark, manufacturer, or otherwise does not necessarily constitute or imply its endorsement, recommendation, or favoring by the United States government or Lawrence Livermore National Security, LLC. The views and opinions of authors expressed herein do not necessarily state or reflect those of the United States government or Lawrence Livermore National Security, LLC, and shall not be used for advertising or product endorsement purposes.

This work performed under the auspices of the U.S. Department of Energy by Lawrence Livermore National Laboratory under Contract DE-AC52-07NA27344. 


\title{
Comparison of observed and theoretical Fe L emission from CIE plasmas
}

\author{
M. Carpenter§, P. Beiersdorfer*, G. V. Brown*, H. C. Chen*, M. F. Gu*, \\ and J. G. Jernigan§
}

*Lawrence Livermore National Laboratory,
$\S$ Space Science Laboratory, Berkeley, CA

\begin{abstract}
We analyze data from the Lawrence Livermore National Lab (LLNL) Electron Beam Ion Trap (EBIT) that simulates a CIE plasma by sweeping the electron beam to approximate a Maxwellian velocity distribution. These results are compared to spectra of confirmed astronomical CIE plasmas (e.g. outer regions of x-ray clusters) observed by XMM/RGS. We utilize the Photon Clean Method (PCM) to quantify these spectra (EBIT and $\mathrm{XMM} / \mathrm{RGS}$ ) in the form of ratios of Fe L lines in the emission complex near $1 \mathrm{keV}$. The variances of line fluxes are measured with bootstrap methods (Efron 1979). Both of these observations are further compared with theoretical predictions of $\mathrm{Fe} L$ line fluxes from APED and similar atomic databases.

Part of this work was performed under the auspices of the U. S. Department of Energy by Lawrence Livermore National Laboratory under Contract W-7405-Eng-48.
\end{abstract}




\title{
Comparison of Observed and Theoretical Fe L Emission from CIE Plasmas
}

\author{
Matthew Carpenter \\ UC Berkeley Space Sciences Laboratory
}

\section{Collaborators}

Lawrence Livermore National Lab Electron Beam Ion Trap (EBIT) Team
P. Beiersdorfer
G. Brown
M. F. Gu
H. Chen

UC Berkeley Space Sciences Laboratory

J. G. Jernigan 
* Introduction to the Photon Clean Method (PCM)

* An Example : XMM/RGS spectrum of Ab Dor

* PCM algorithm internals

* Analysis Modes: Phase I and Phase II solutions

* Bootstrap Methods of error analysis

* Summary 


\section{Photon Clean Method: Principles}

- Analysis uses individual photon events, not binned spectra

- Fitting models to data is achieved through weighted random trial-anderror with feedback

- Individual photons span parameter and model space, and are taken to be the parameters

- Iteration until quantitative convergence based on a Kolmogorov-Smirnov (KS) test

- Has analysis modes which allow divergence from strict adherence to model to estimate differences between model and observed data 


\section{Photon Clean Method: Event-Mode Data and Model}

Both data and model are in form of Event Lists (photon lists)

Monte-Carlo methods are used to generate simulated photons

Generation parameters for each photon are recorded
Each photon is treated as independent parameter

Single simulated photon:

\begin{tabular}{cccccc}
$\lambda_{\text {spec }}(\AA)$ & $\lambda_{\text {sim }}(\AA)$ & $\log \left(T_{M K}\right)$ & $T_{k e V}$ & Type & Transition \\
\hline 18.990 & 18.967 & 6.796 & 0.538 & O VIII & $2 p^{2} P_{3 / 2} \rightarrow 1 s^{2} S_{1 / 2}$
\end{tabular}




\section{PCM analyzes Simulated Detected $\lambda_{\text {sim }}$ or $\mathrm{E}_{\text {sim }}$}

\begin{tabular}{|c|c|}
\hline $\begin{array}{l}\text { Observed Data } \\
\text { (Event Form) } \\
\lambda_{\text {observed }}(\AA)\end{array}$ & $\begin{array}{c}\text { Simulatec } \\
\text { Data } \\
\lambda_{\text {sim }}(\AA)\end{array}$ \\
\hline 15.020 & 18.990 \\
\hline 14.961 & 12.290 \\
\hline 7.622 & 10.761 \\
\hline 17.711 & 12.807 \\
\hline 21.549 & 17.930 \\
\hline 16.062 & 12.238 \\
\hline 14.376 & 13.470 \\
\hline 13.298 & 11.510 \\
\hline 12.833 & 28.279 \\
\hline 17.801 & 13.105 \\
\hline 21.217 & 17.037 \\
\hline
\end{tabular}

Data representation inside program

Target: AB Dor (K1 IV-V), a young active star and XMM/RGS calibration target 


\section{PCM analyzes Simulated Detected $\lambda_{\text {sim }}$ or $\mathrm{E}_{\text {sim }}$}

The Photon Clean Method algorithm analyzes and outputs models as event lists *** All histograms in this talk are for visualization only

\begin{tabular}{rr}
$\lambda_{\text {observed }}(\AA)$ & $\lambda_{\text {sim }}(\AA)$ \\
\hline 15.020 & 18.990 \\
14.961 & 12.290 \\
7.622 & 10.761 \\
17.711 & 12.807 \\
21.549 & 17.930 \\
16.062 & 12.238 \\
14.376 & 13.470 \\
13.298 & 11.510 \\
12.833 & 28.279 \\
17.801 & 13.105 \\
21.217 & 17.037 \\
$\vdots$ & $\vdots$
\end{tabular}

star and XMM/RGS calibration target

active ( 


\section{PCM analyzes Simulated Detected $\lambda_{\text {sim }}$ or $\mathrm{E}_{\text {sim }}$}

Target: AB Dor (K1 IV-V), a young active star and XMM/RGS calibration target

\begin{tabular}{rc}
$\lambda_{\text {observed }}(\AA)$ & $\lambda_{\text {sim }}(\AA)$ \\
\hline 15.020 & 18.990 \\
14.961 & 12.290 \\
7.622 & 10.761 \\
17.711 & 12.807 \\
21.549 & 17.930 \\
16.062 & 12.238 \\
14.376 & 13.470 \\
13.298 & 11.510 \\
12.833 & 28.279 \\
17.801 & 13.105 \\
21.217 & 17.037 \\
$\vdots$ & $\vdots$
\end{tabular}

The Photon Clean Method algorithm analyzes and outputs models as event lists *** All histograms in this talk are for visualization only

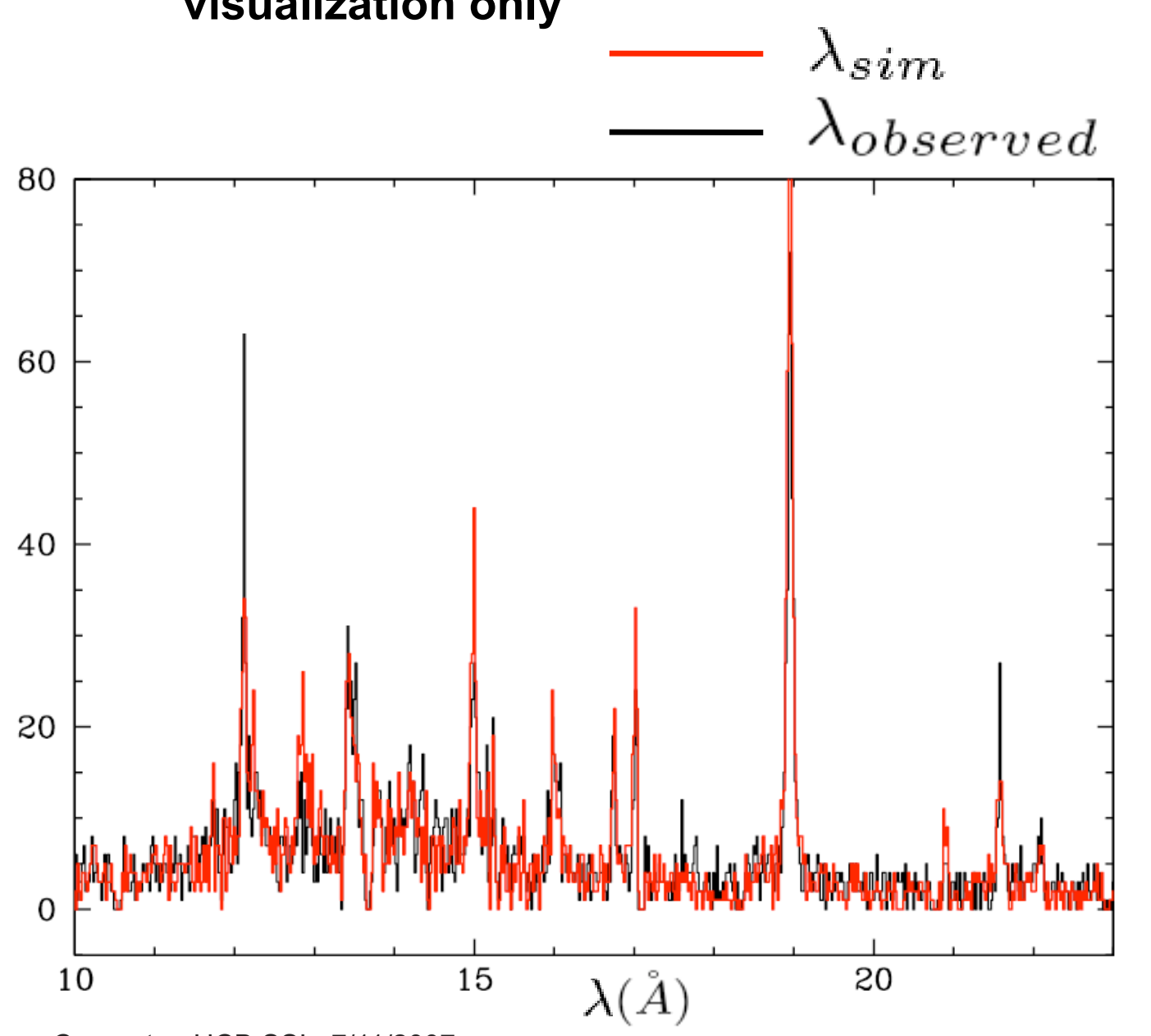

Matthew Carpenter, UCB SSL, 7/11/2007 
Each photon in a simulated observation has ideal (model) wavelength and the wavelength of detection

$\lambda_{\text {spec }}==$ "spectral wavelength" from plasma model

\begin{tabular}{ccccl} 
Phot.\# & $\lambda_{\text {spec }}(\AA)$ & $\lambda_{\text {sim }}(\AA)$ & $\log \left(T_{M K}\right)$ & \multicolumn{1}{c}{ Type } \\
\hline 1 & 18.990 & 18.967 & 6.796 & O VIII \\
2 & 12.903 & 12.308 & 6.839 & Continuum \\
3 & 10.762 & 10.849 & 7.096 & Ni XXIII \\
4 & 12.806 & 12.846 & 7.129 & Fe XX \\
5 & 17.930 & 18.037 & 6.662 & Continuum \\
6 & 12.238 & 12.284 & 7.030 & Fe XXI \\
$\vdots$ & $\vdots$ & $\vdots$ & $\vdots$ & $\vdots$
\end{tabular}


Each photon in a simulated observation has ideal (model) wavelength and the wavelength of detection

$\lambda_{\text {spec }}==$ "spectral wavelength" from plasma model

A histogram of the simulated photons' spectral wavelengths produces sharp lines

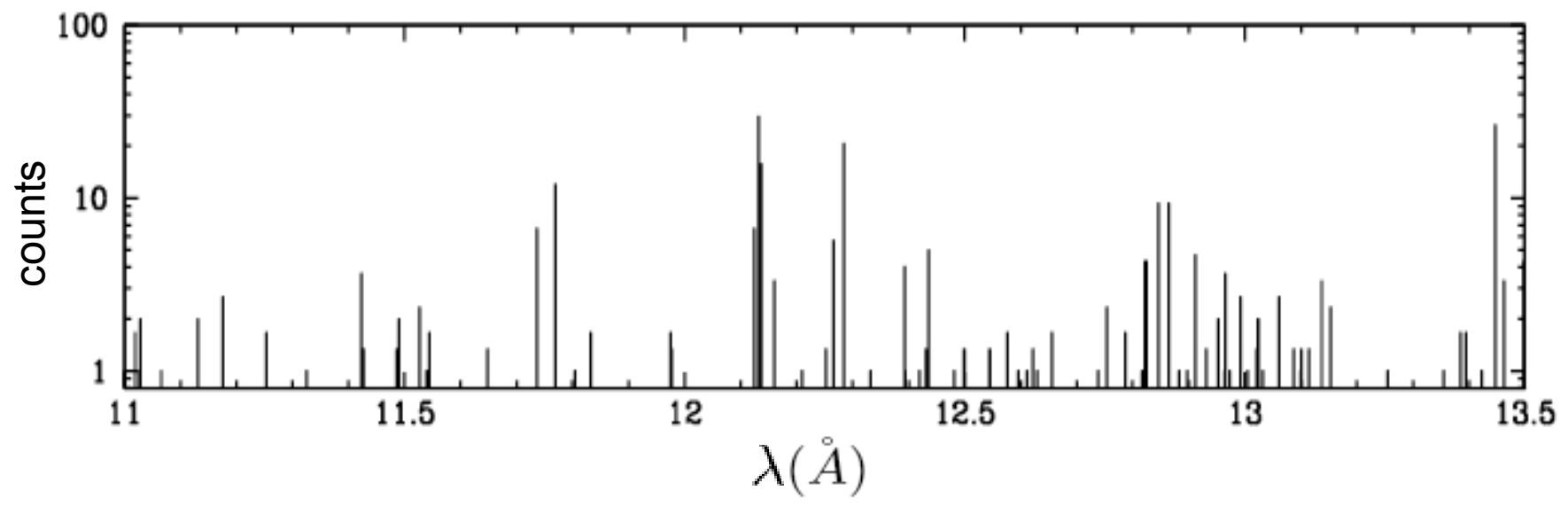

Type

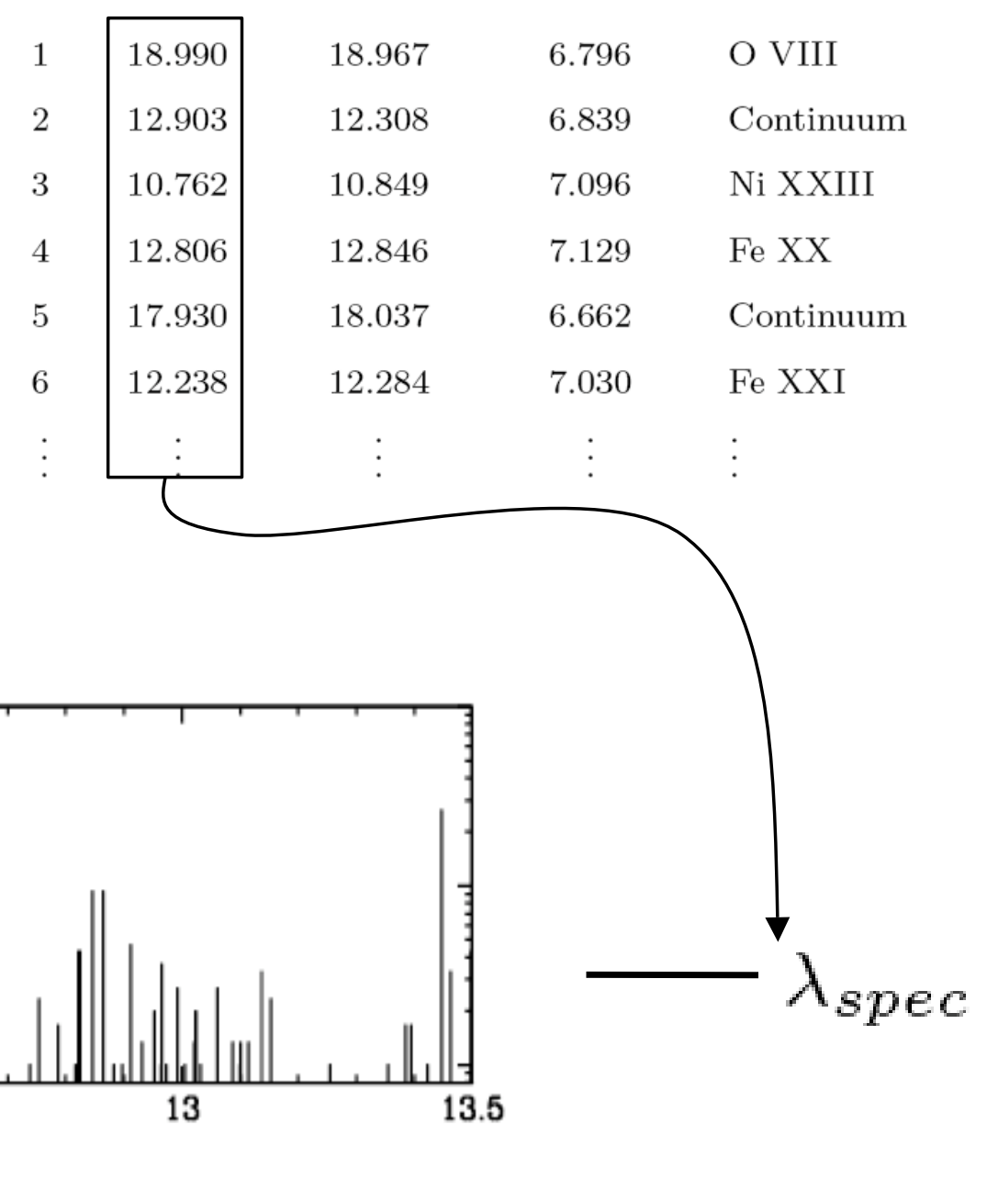




\section{Adding detector response}

Photons are stochastically assigned a "detected wavelength"

$\lambda_{\text {sim }}==$ "simulated wavelength," includes redshift, detector and thermal

\section{broadening}

\begin{tabular}{|c|c|c|c|c|}
\hline Phot.\# & $\lambda_{\text {spec }}(\AA)$ & $\lambda_{\text {sim }}(\AA)$ & $\log \left(T_{M K}\right)$ & Type \\
\hline 1 & 18.990 & 18.967 & 6.796 & O VIII \\
\hline 2 & 12.903 & 12.308 & 6.839 & Continuum \\
\hline 3 & 10.762 & 10.849 & 7.096 & Ni XXIII \\
\hline 4 & 12.806 & 12.846 & 7.129 & Fe XX \\
\hline 5 & 17.930 & 18.037 & 6.662 & Continuum \\
\hline 6 & 12.238 & 12.284 & 7.030 & Fe XXI \\
\hline
\end{tabular}

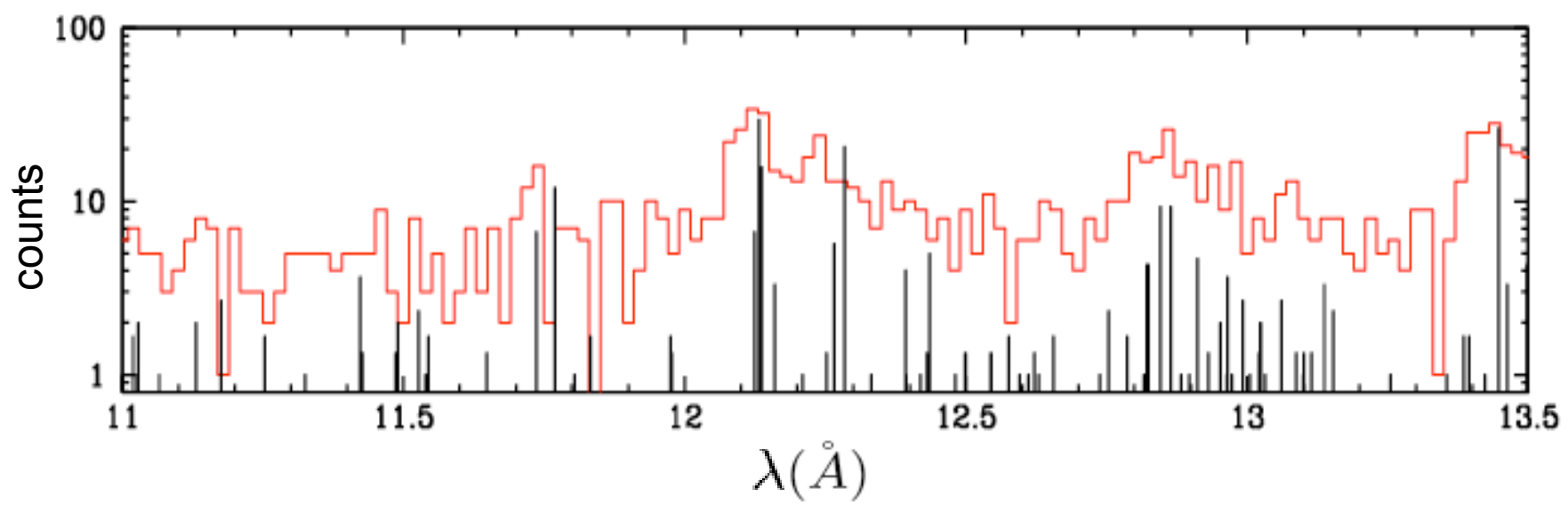




\section{Distribution of Model Parameters}

Each photon has individual parameter values which may be taken as elements of the parameter distribution

$$
\text { Phot.\# }
$$

$\lambda_{\text {spec }}(\stackrel{\circ}{A})$

$\lambda_{\operatorname{sim}}(\AA$

$\log (\mathrm{T})$

Type

\begin{tabular}{ccc|c|l}
1 & 18.990 & 18.967 & 6.796 & O VIII \\
2 & 12.903 & 12.308 & 6.839 & Continuum \\
3 & 10.762 & 10.849 & 7.096 & Ni XXIII \\
4 & 12.806 & 12.846 & 7.129 & Fe XX \\
5 & 17.930 & 18.037 & 6.662 & Continuum \\
6 & 12.238 & 12.284 & 7.030 & Fe XXI \\
$\vdots$ & $\vdots$ & $\vdots$ & $\vdots$ & $\vdots$
\end{tabular}




\section{Distribution of Model Parameters}

Each photon has individual parameter values which may be taken as elements of the parameter distribution

The temperature profile of $A B$ Dor is complex; previous fits used 3-temperature or EMD models

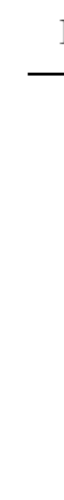

Phot.\#

$\lambda_{\text {spec }}(\stackrel{\circ}{)}$

\begin{tabular}{lll|l|l}
1 & 18.990 & 18.967 & 6.796 & O VIII \\
2 & 12.903 & 12.308 & 6.839 & Continuum \\
3 & 10.762 & 10.849 & 7.096 & Ni XXIII \\
4 & 12.806 & 12.846 & 7.129 & Fe XX \\
5 & 17.930 & 18.037 & 6.662 & Continuum \\
6 & 12.238 & 12.284 & 7.030 & Fe XXI
\end{tabular}

AB Dor Emission Measure Distribution

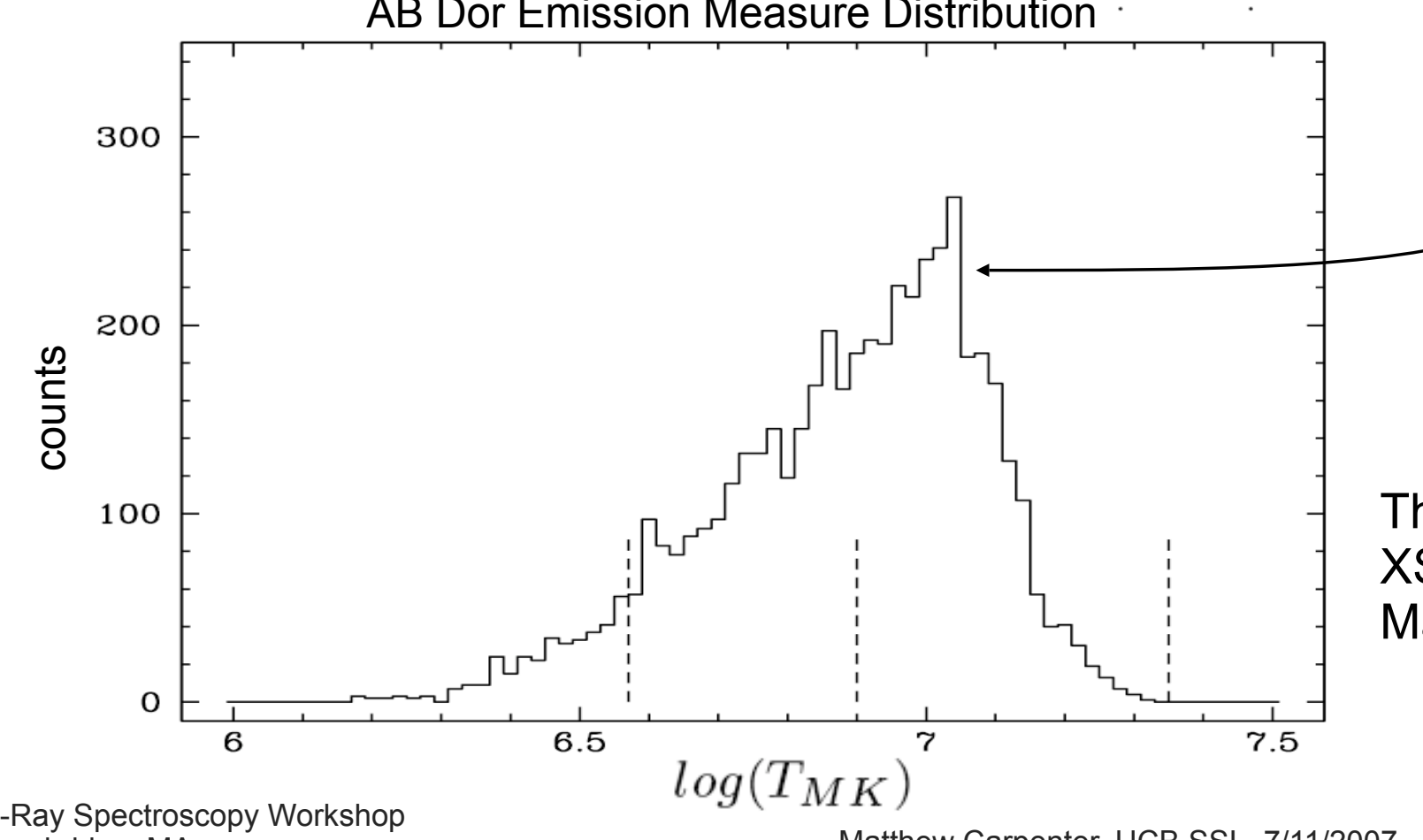

Three vertical dashed lines are 3-T XSPEC fit from Sanz-Forcada, Maggio and Micela (2003) 
Start: Generate initial model + simulated detected photons from input parameter distribution

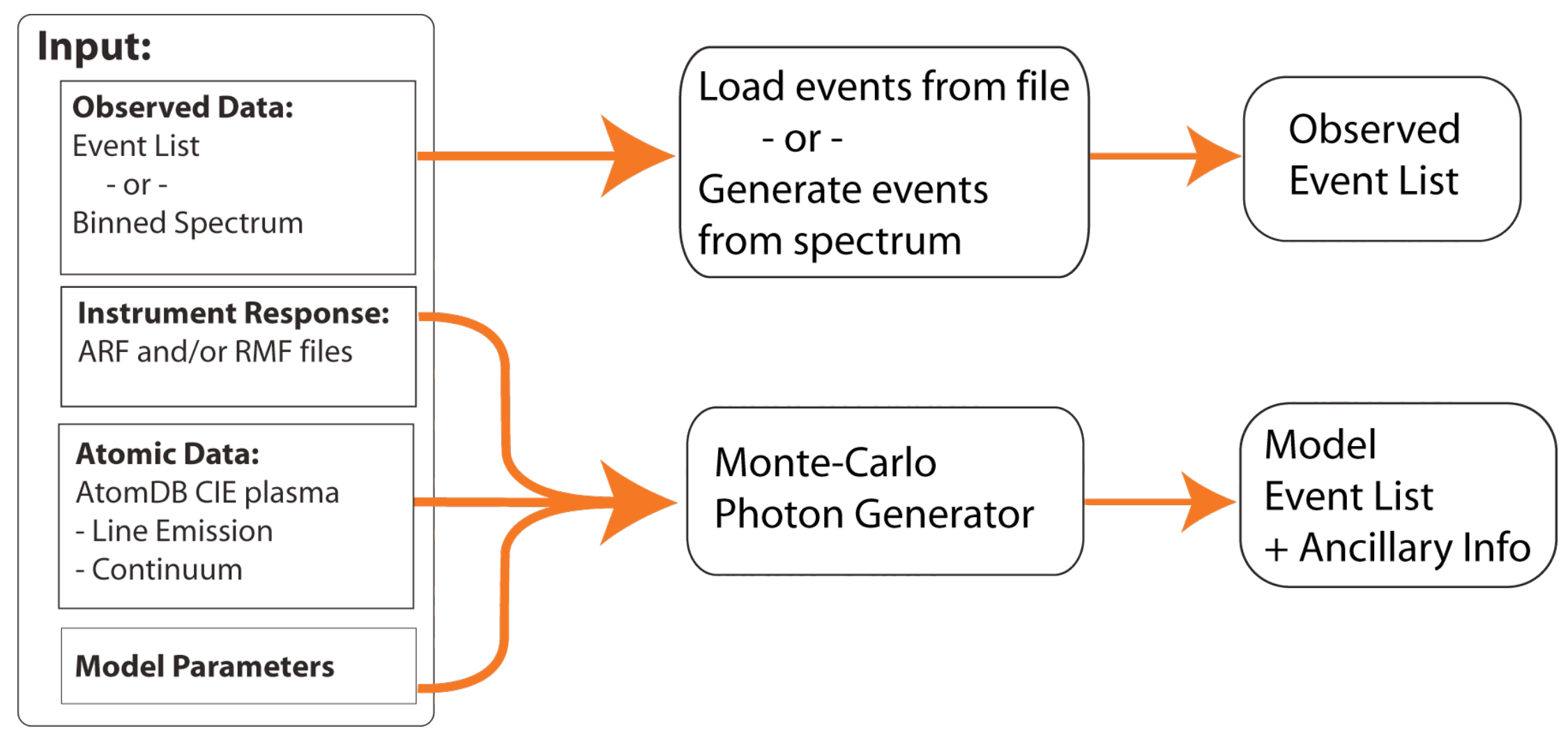


Start: Generate initial model + simulated detected photons from input parameter distribution

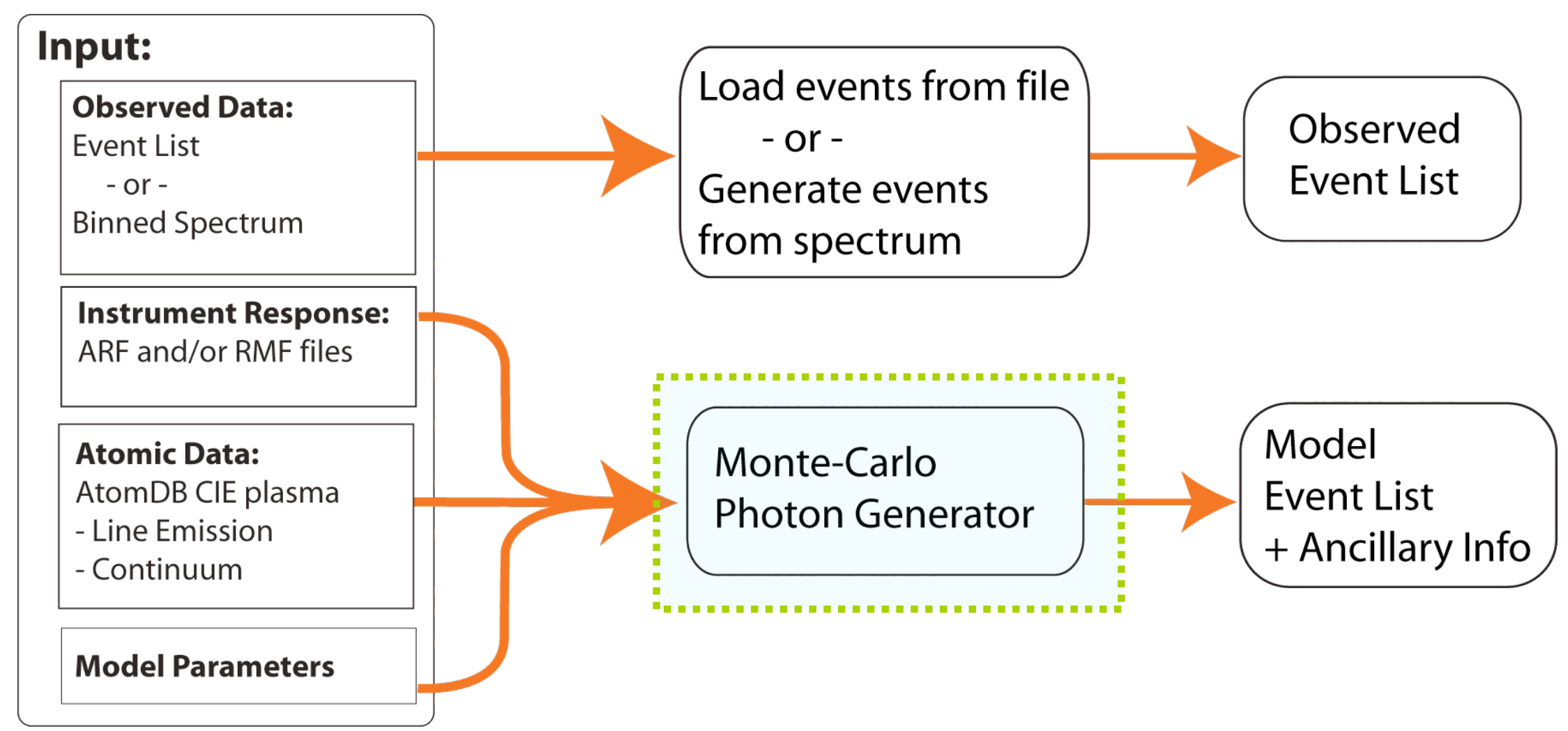




\section{Photon Generator}

\section{Start: Model Parameter $(\mathrm{T})$}

\section{For CIE Plasma:}

- Given a temperature ( $T$ ), AtomDB generates spectral energy $(E)$.

-Apply ARF test to determine whether photon is detected

- If photon is detected, apply RMF to determine detected energy $\left(E^{\prime}\right)$

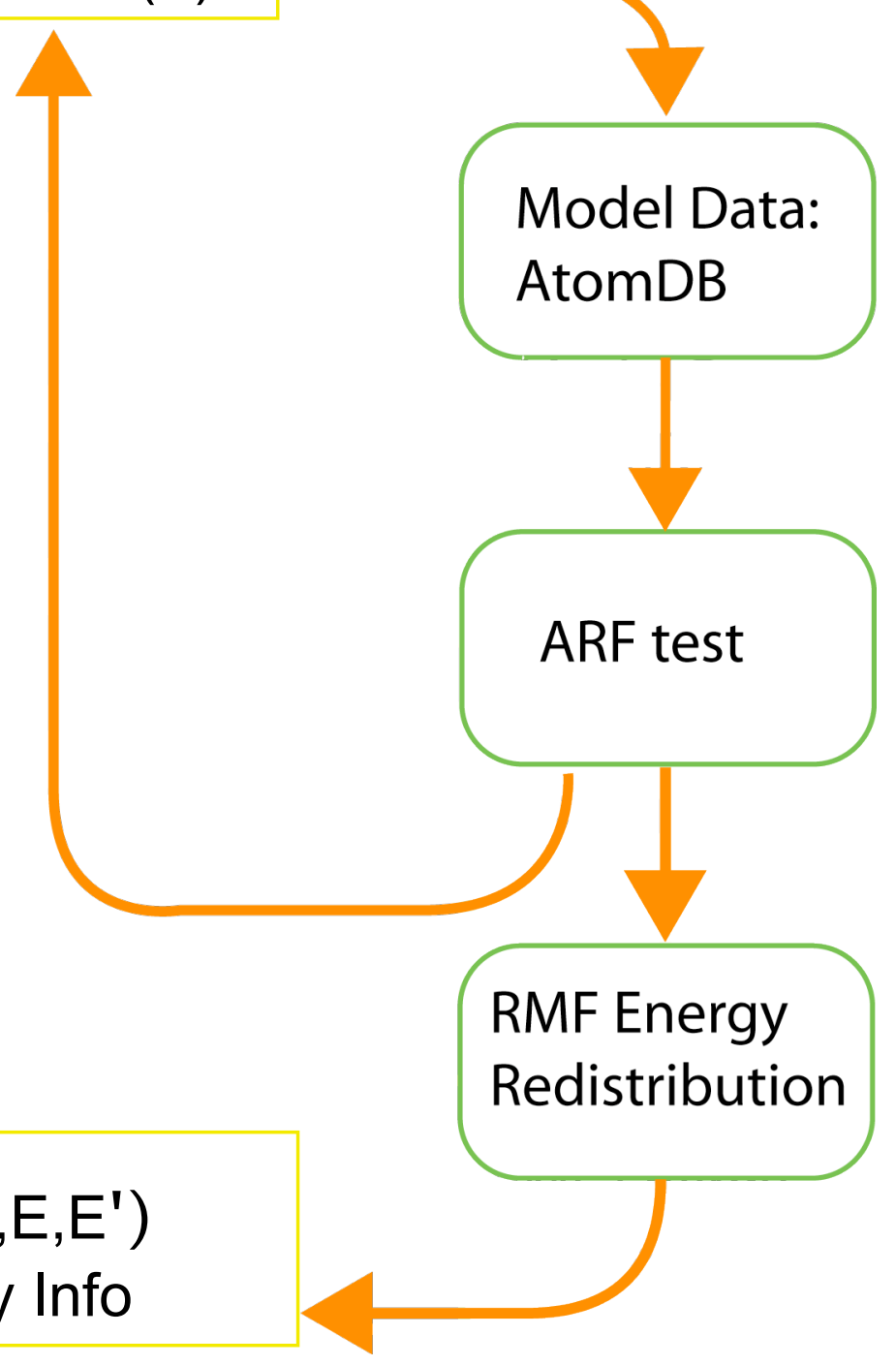

Result: (T,E,E')

+ Ancillary Info 


\section{PCM Algorithm: Iterate with Feedback}

Model $\mathrm{E}_{\text {sim }}$

Iteration: 


\section{PCM Algorithm: Iterate with Feedback}

Model $\mathrm{E}_{\text {sim }}$

Iteration:

- Generate 1 detected photon

- Replace 1 random photon from model with new photon $\left(E, E^{\prime}, T\right)$

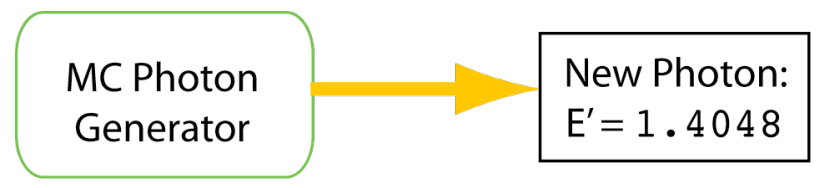

\begin{tabular}{|c|}
\hline$\vdots$ \\
2.4903 \\
1.6309 \\
0.9901 \\
1.4052 \\
1.1385 \\
1.6027 \\
3.7938 \\
2.4429 \\
$\vdots$ \\
\hline
\end{tabular}


Model $\mathrm{E}_{\text {sim }}$

Iteration:

- Generate 1 detected photon

- Replace 1 random photon from model with new photon $\left(E, E^{\prime}, T\right)$

- Compute KS probability statistic
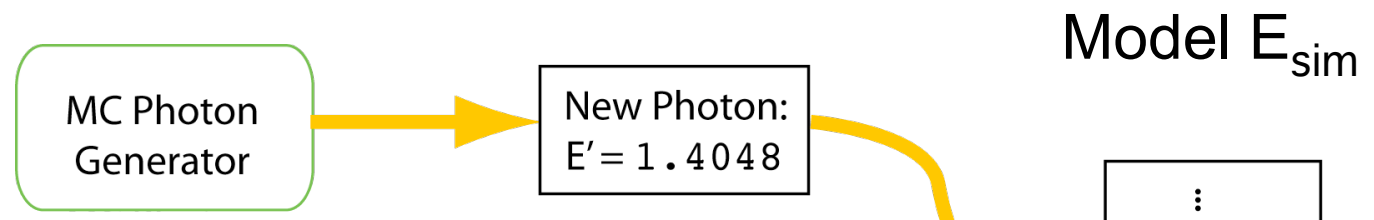

\section{Feedback Test:}

If KS probability improves with

new photon, keep it;

Otherwise, throw new photon away

and keep old photon

KS Statistic:

$\mathrm{D}=0.0834$

Prob $=1.2 \mathrm{e}-8$
KS Test:

Compare Observed E with Model E' 


\section{PCM Analysis Modes}

\section{Phase I: Constrained Convergence}

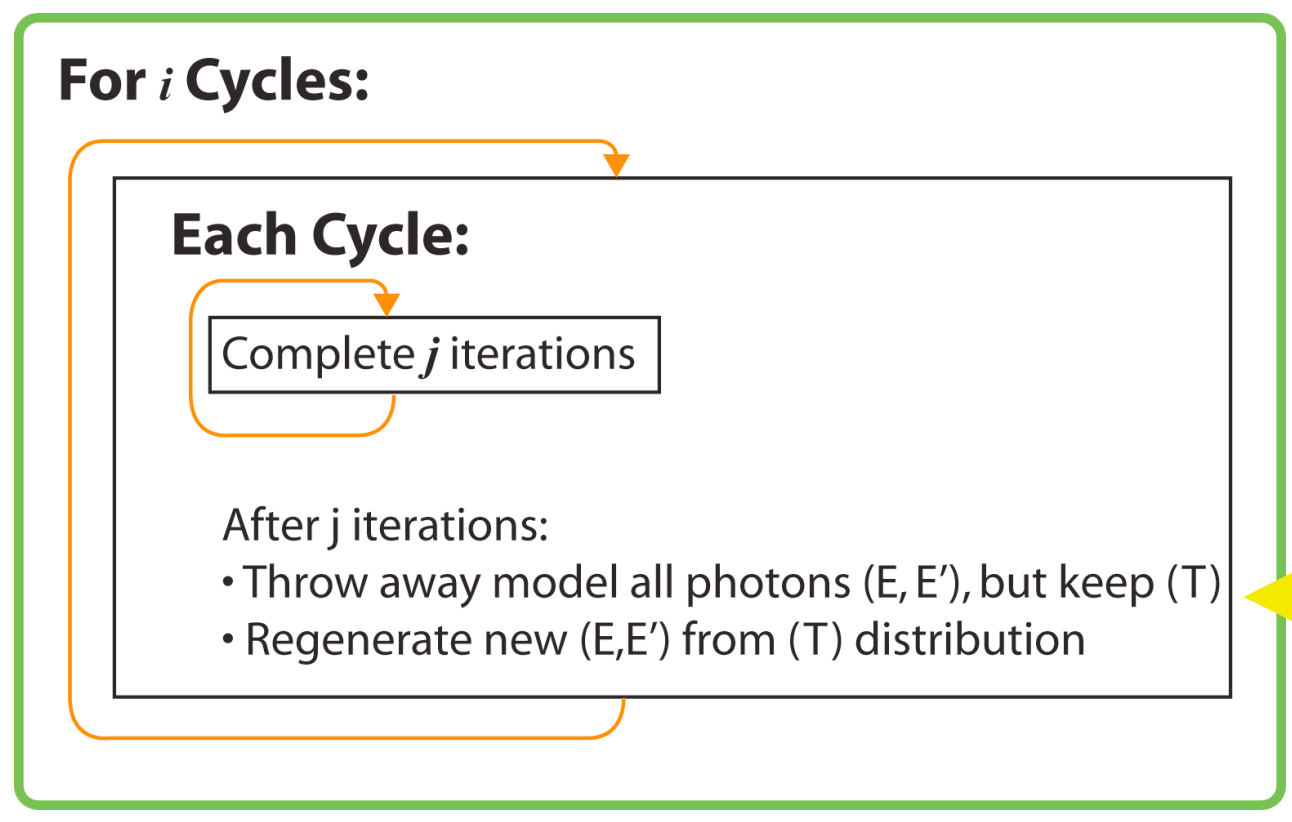

"Re-constrain the Model"

- Generates a solution which is consistent with a physically realizable model 


\section{PCM Analysis Modes}

Phase II: Un-Constrained Convergence:

- Iterate until KS probability reaches cutoff value, with Monte-Carlo Markov Chain weighting

- Photon distribution is not constrained to model probabilities

- Allows individual spectral features to be modified to produce bestfit solution

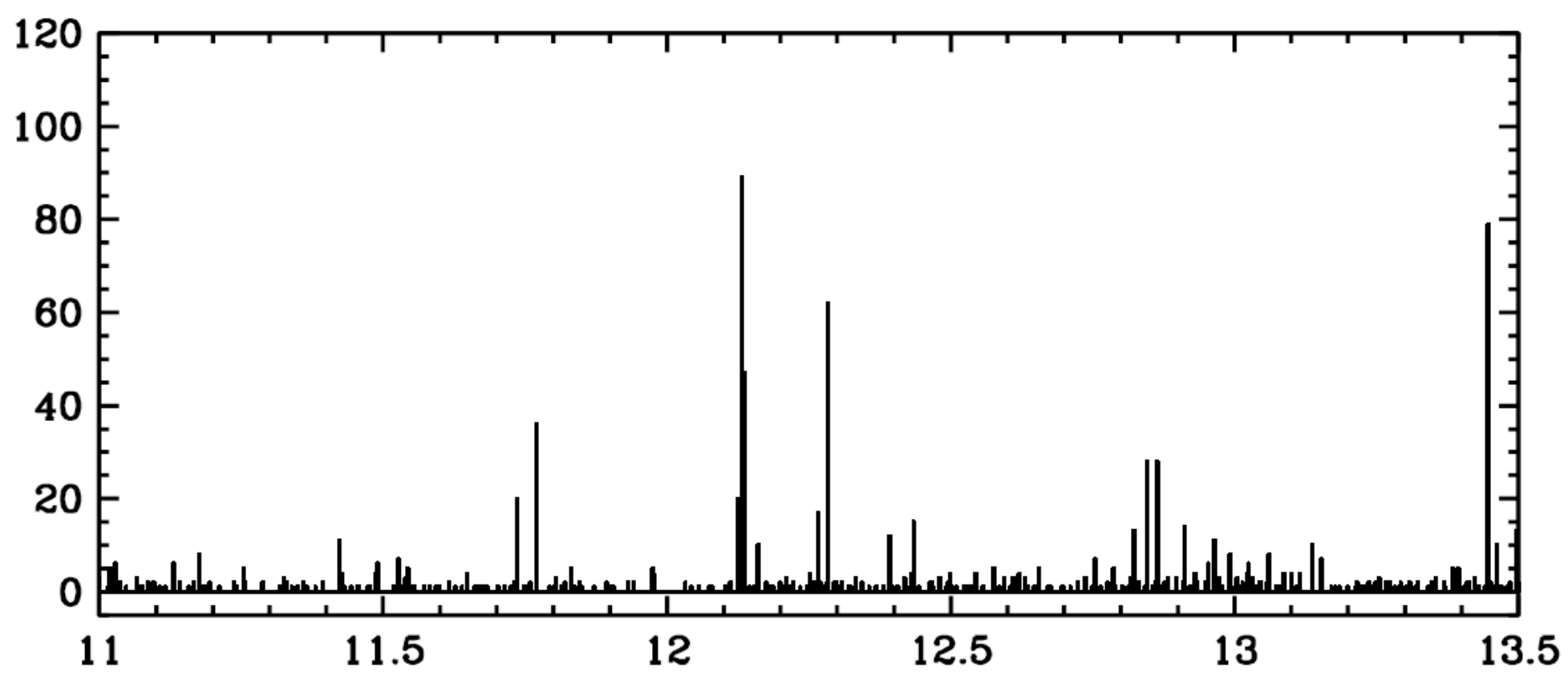




\section{PCM Analysis Modes}

Phase II: Un-Constrained Convergence:

- Iterate until KS probability reaches cutoff value, with Monte-Carlo Markov Chain weighting

- Photon distribution is not constrained to model probabilities

- Allows individual spectral features to be modified to produce bestfit solution

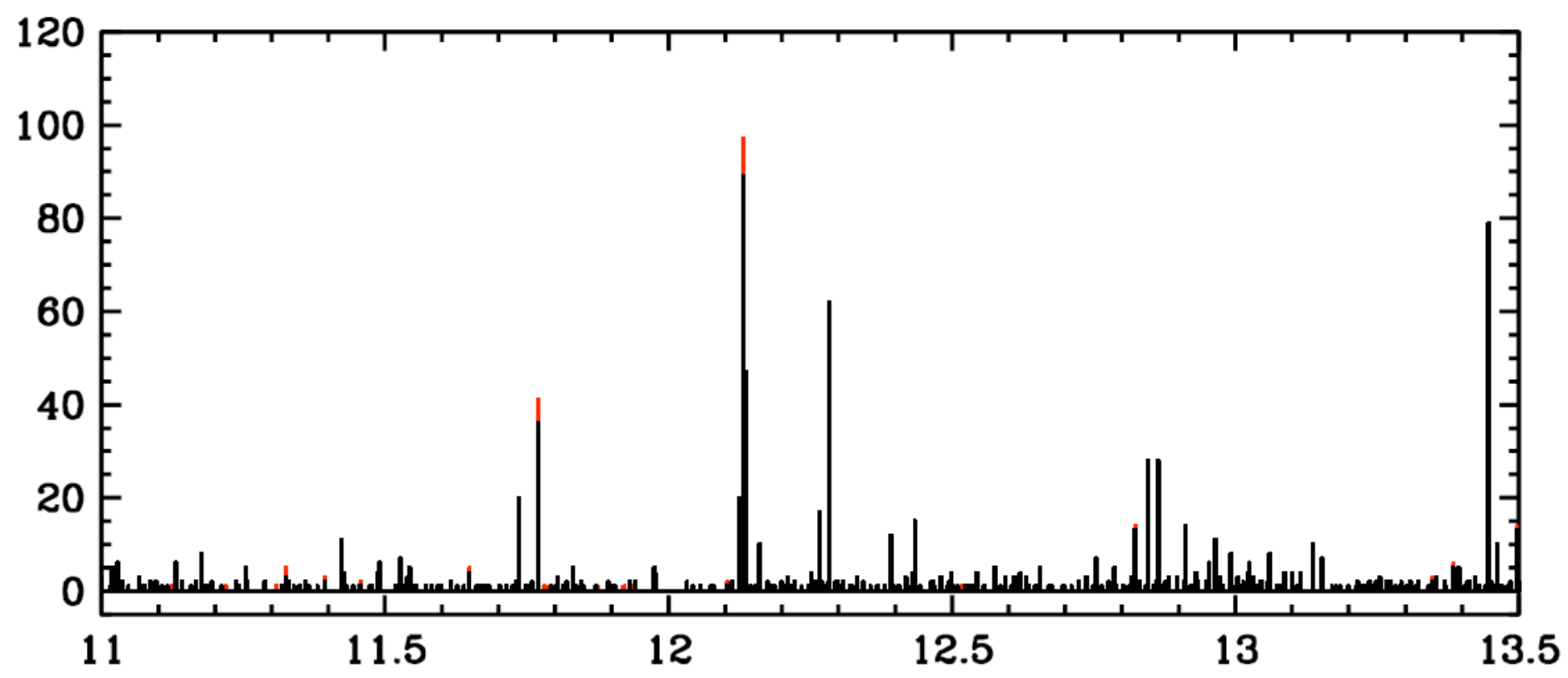




\section{Determining Variation in Many-Parameter Models}

Low-dimensionality models with few degrees of freedom may be quantified using Chi-square test which has a well-defined error methodology. PCM is appropriate for models of high dimensionality where every photon is a free parameter.

For error determination we use distribution-driven re-sampling methods

$\Rightarrow$ Bootstrap Method

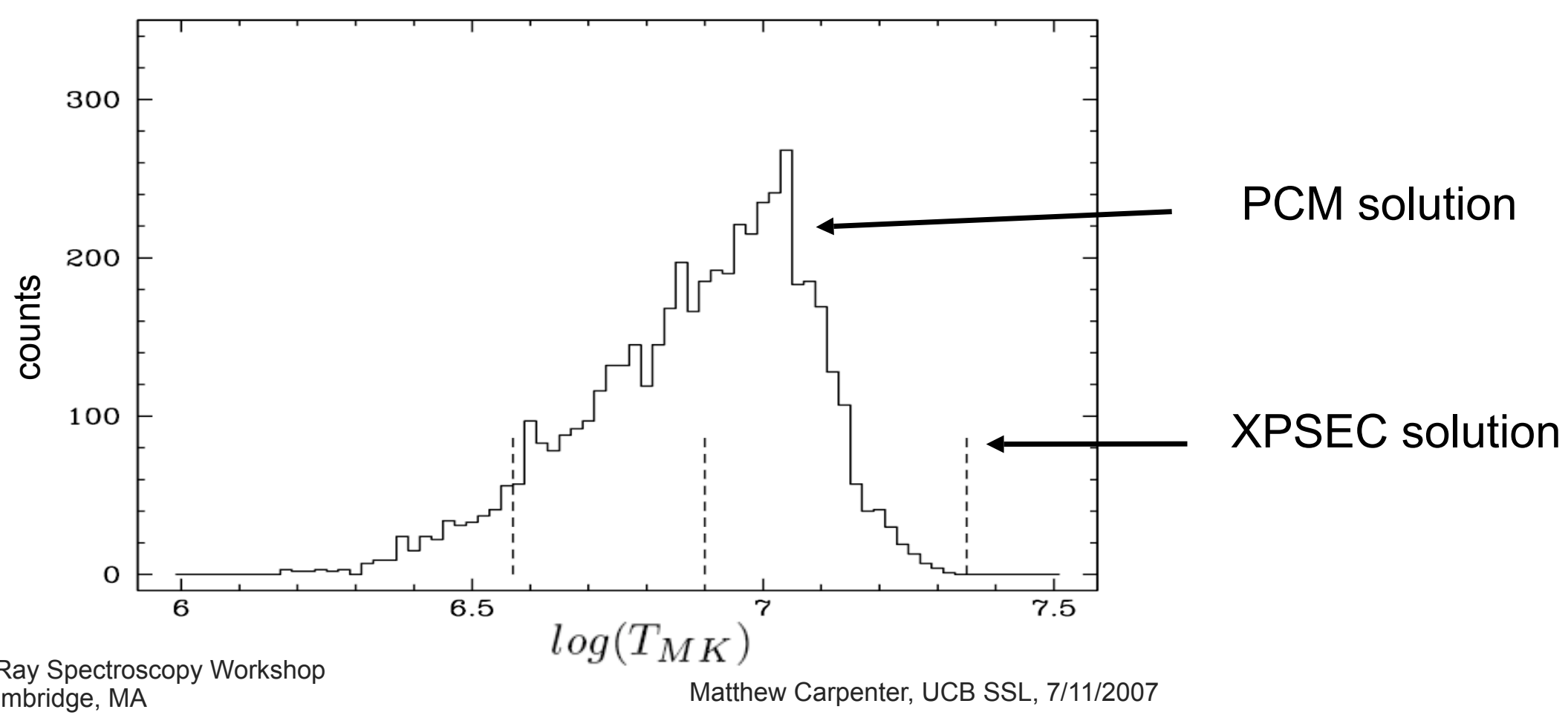




\section{Bootstrap Re-Sampling}

Method:

1) Randomly resample input data set with substitution to create new data set

2) Perform analysis on new data set to produce new outcome

3) Repeat for $n>>1$ re-sampled data sets 


\section{Bootstrap Re-Sampling}

Method:

1) Randomly resample input data set with substitution to create new data set

2) Perform analysis on new data set to produce new outcome

3) Repeat for $n>>1$ re-sampled data sets

$$
\begin{array}{r}
15.020 \\
14.961 \\
7.622 \\
17.711 \\
21.549 \\
16.062 \\
14.376 \\
13.298 \\
12.833 \\
17.801
\end{array}
$$




\section{Bootstrap Re-Sampling}

Method:

1) Randomly resample input data set with substitution to create new data set

2) Perform analysis on new data set to produce new outcome

3) Repeat for $n>>1$ re-sampled data sets

\begin{tabular}{|r|}
\hline 15.020 \\
\hline 14.961 \\
\hline 7.622 \\
\hline 17.711 \\
\hline 21.549 \\
\hline 16.062 \\
\hline 14.376 \\
\hline 13.298 \\
12.833 \\
\hline 17.801 \\
\hline
\end{tabular}




\section{Bootstrap Re-Sampling}

Method:

1) Randomly resample input data set with substitution to create new data set

2) Perform analysis on new data set to produce new outcome

3) Repeat for $n>>1$ re-sampled data sets

\begin{tabular}{|r|r|}
\hline 15.020 & 15.020 \\
\hline 14.961 & 7.622 \\
7.622 \\
\hline 7.622 \\
\hline 17.711 \\
\hline 21.549 \\
\hline 16.062 & 17.711 \\
\hline 14.376 & 21.549 \\
\hline 13.298 & 16.062 \\
12.833 & 14.376 \\
\hline 17.801 & 14.376 \\
14.376 \\
\hline
\end{tabular}




\section{Bootstrap Results}

AB Dor Emission Measure Distribution

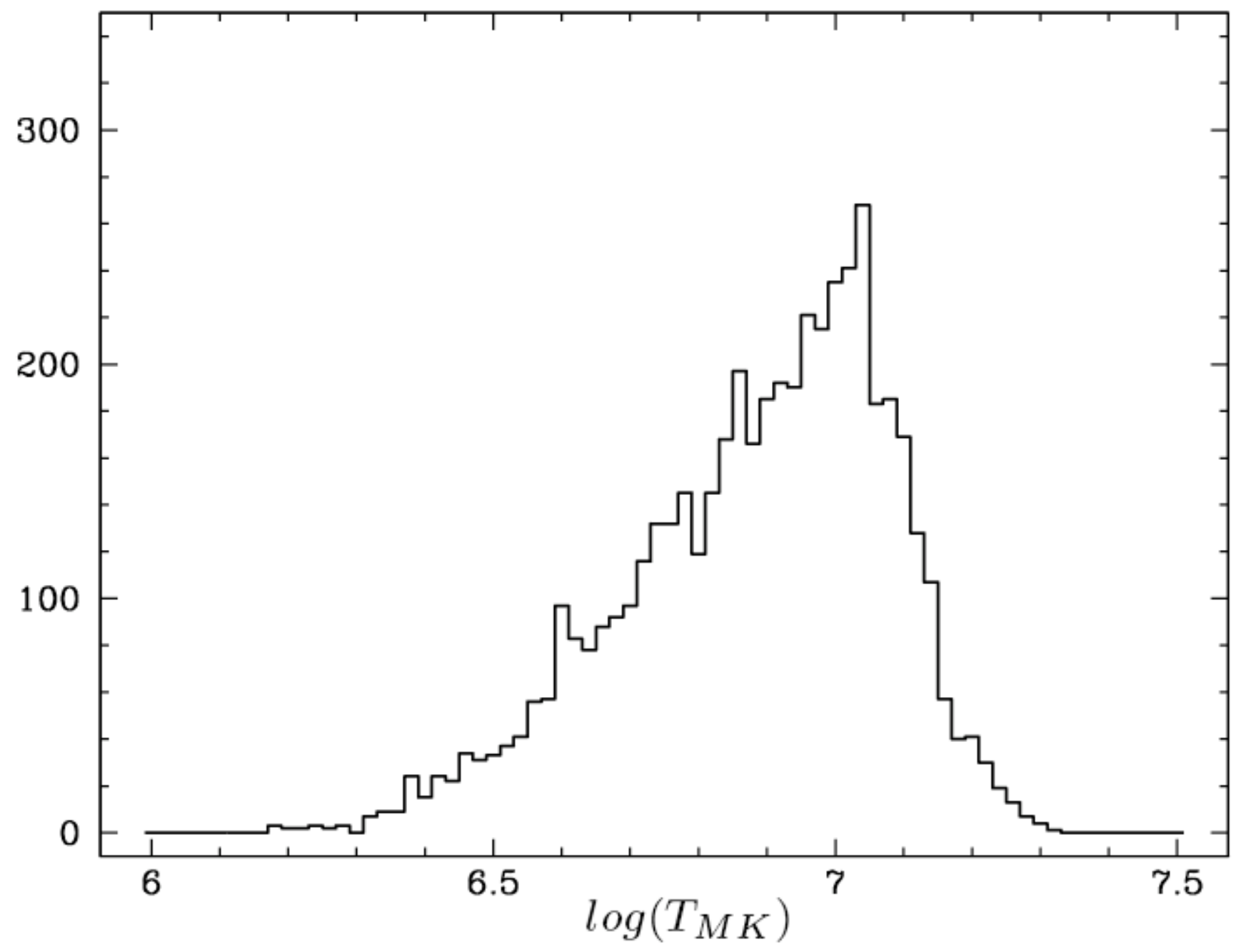




\section{Bootstrap Results}

AB Dor Emission Measure Distribution

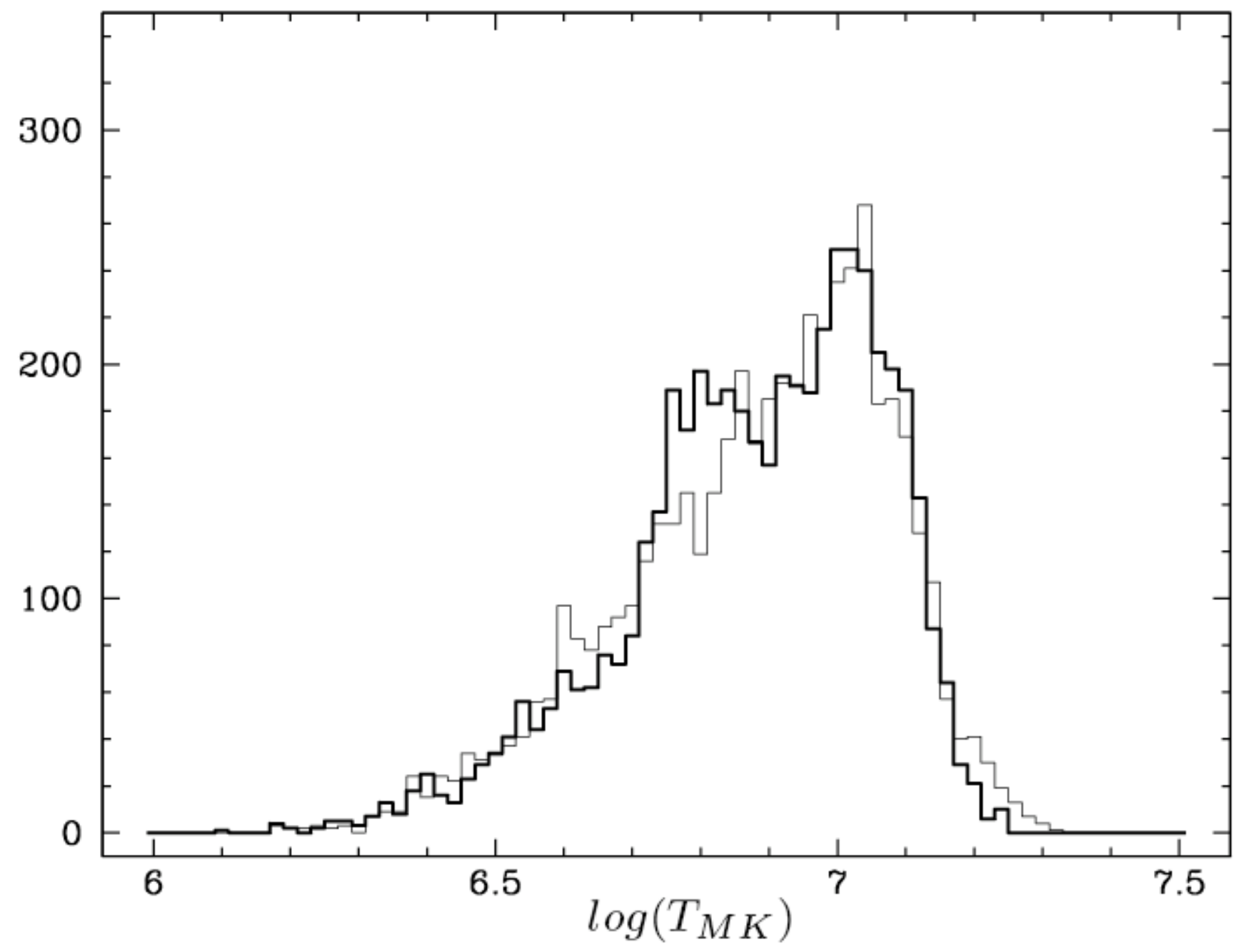




\section{Bootstrap Results}

AB Dor Emission Measure Distribution

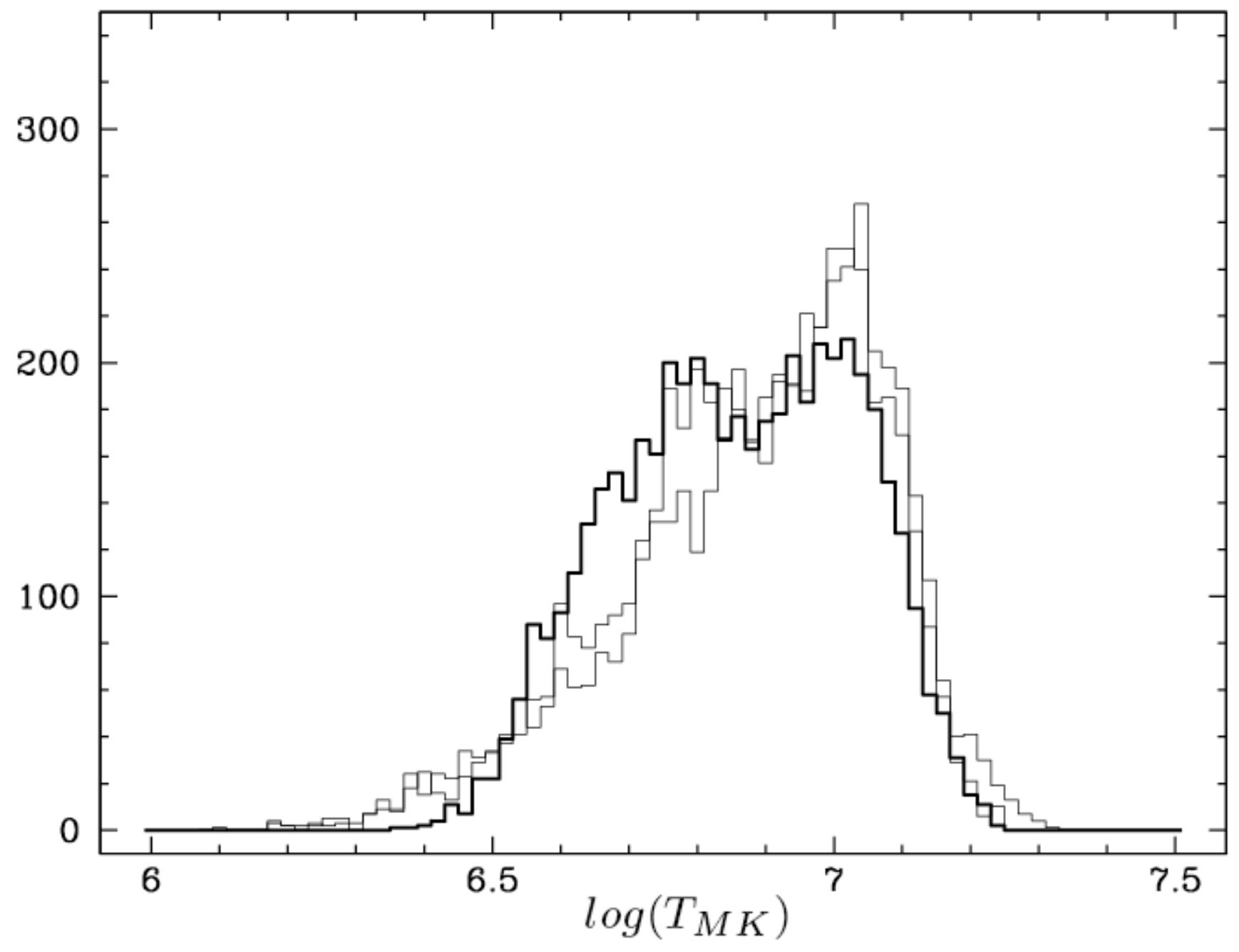




\section{Bootstrap Results}

AB Dor Emission Measure Distribution

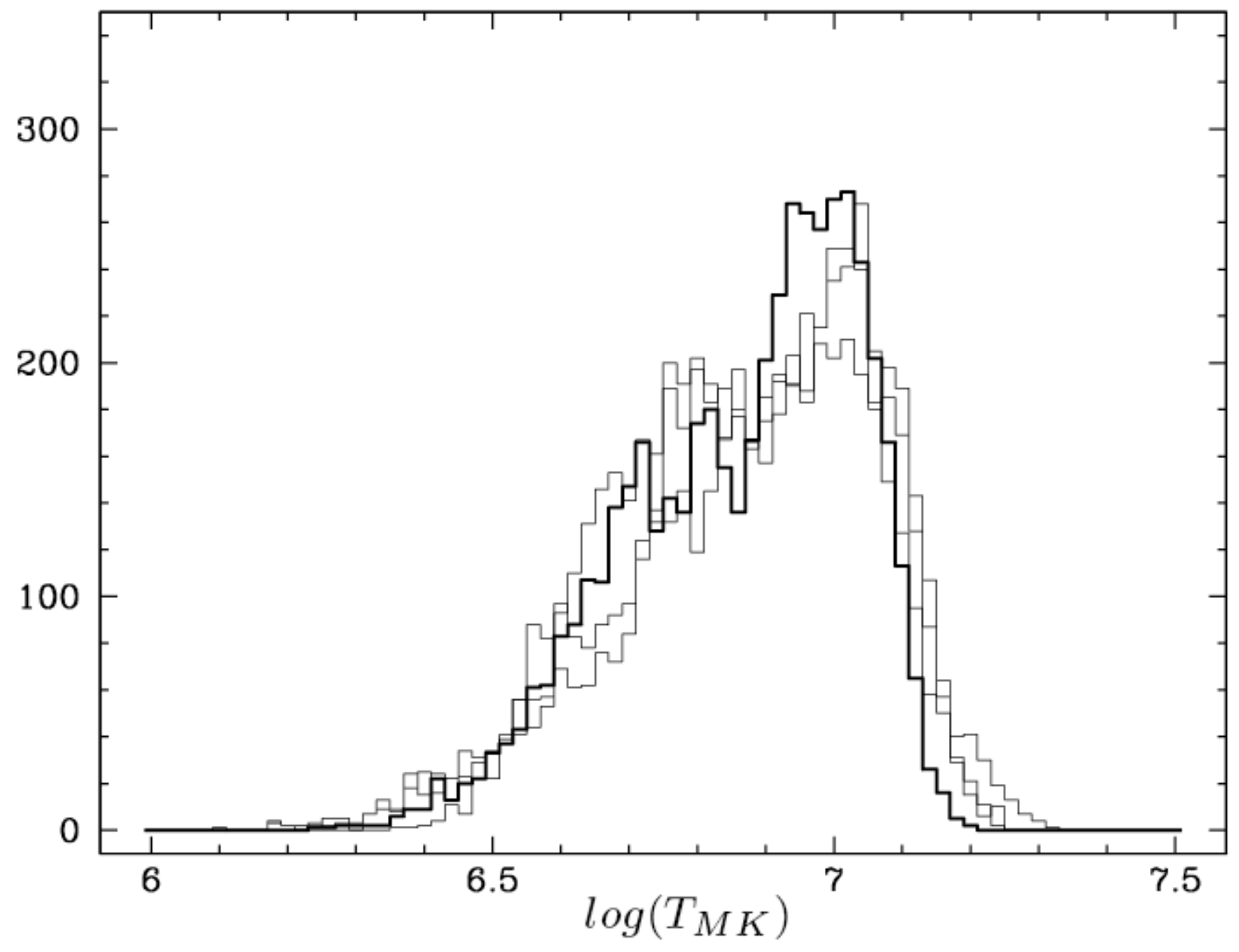




\section{Bootstrap Results}

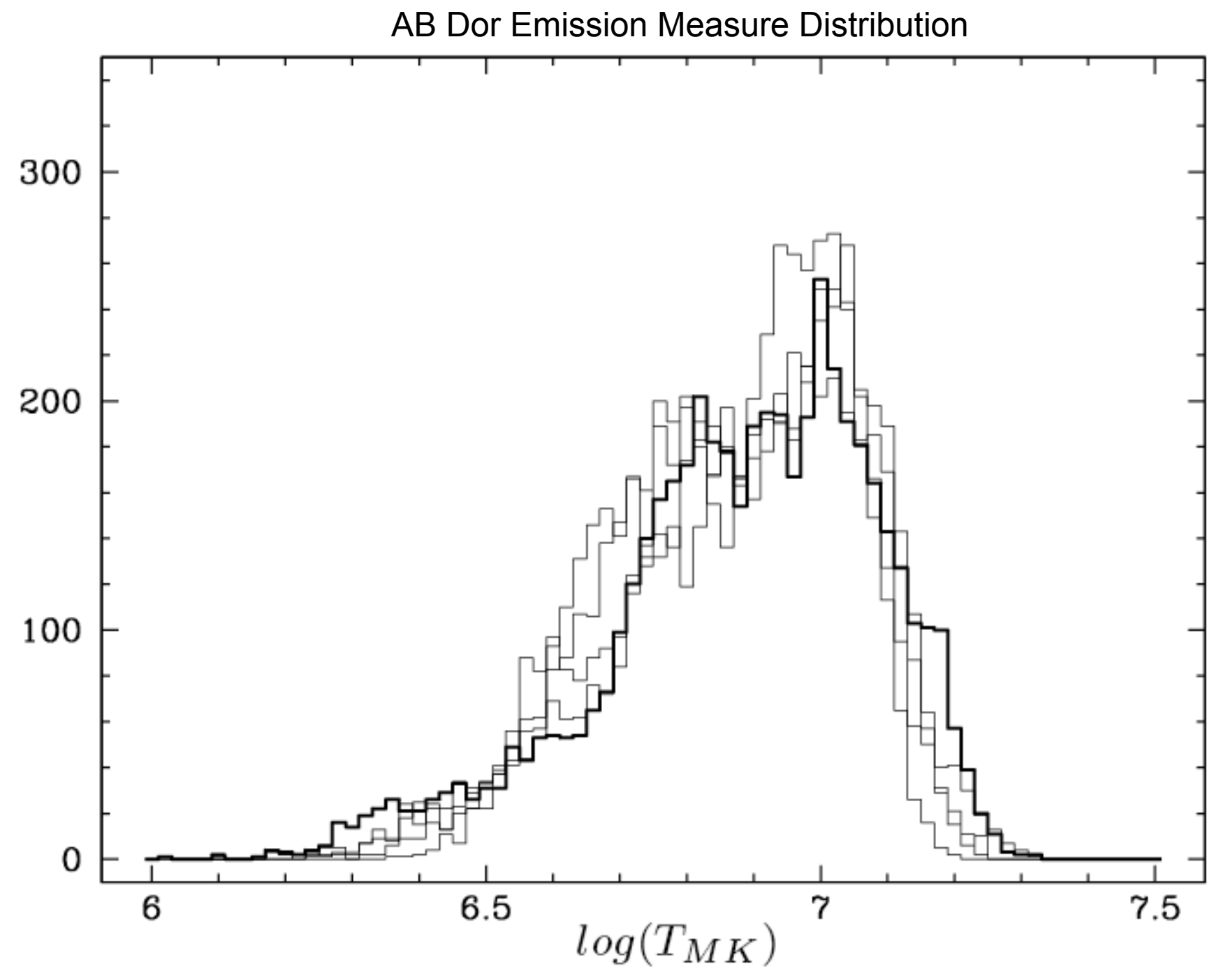




\section{Interpreting the Bootstrap}

The variations in the bootstrap solutions estimate errors

The Arithmetic mean of all distributions is plotted as solid line
AB Dor Emission Measure Distribution

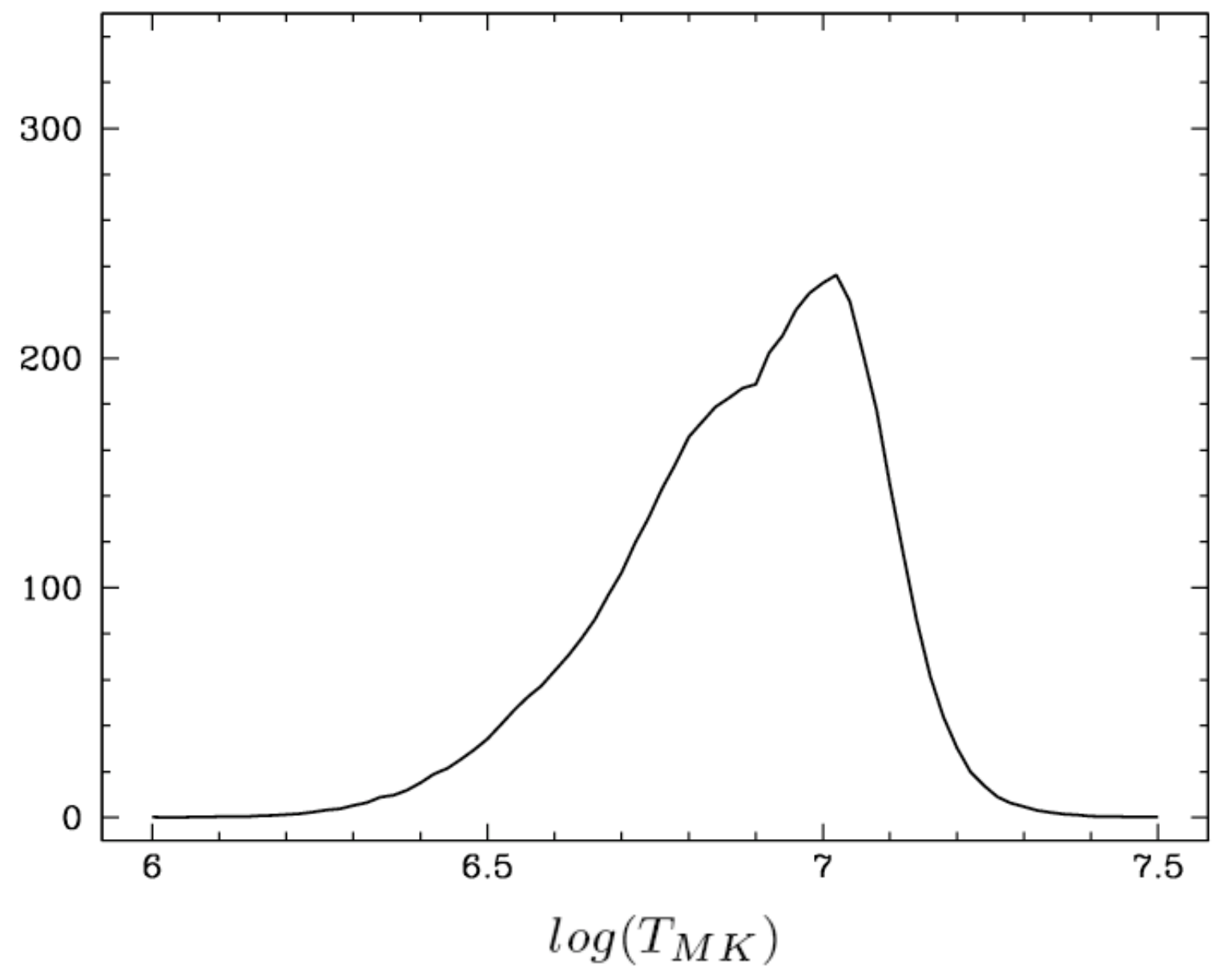




\section{Interpreting the Bootstrap}

The variations in the bootstrap solutions estimate errors

The Arithmetic mean of all distributions is plotted as solid line

Confidence levels are computed along vertical axis of distribution

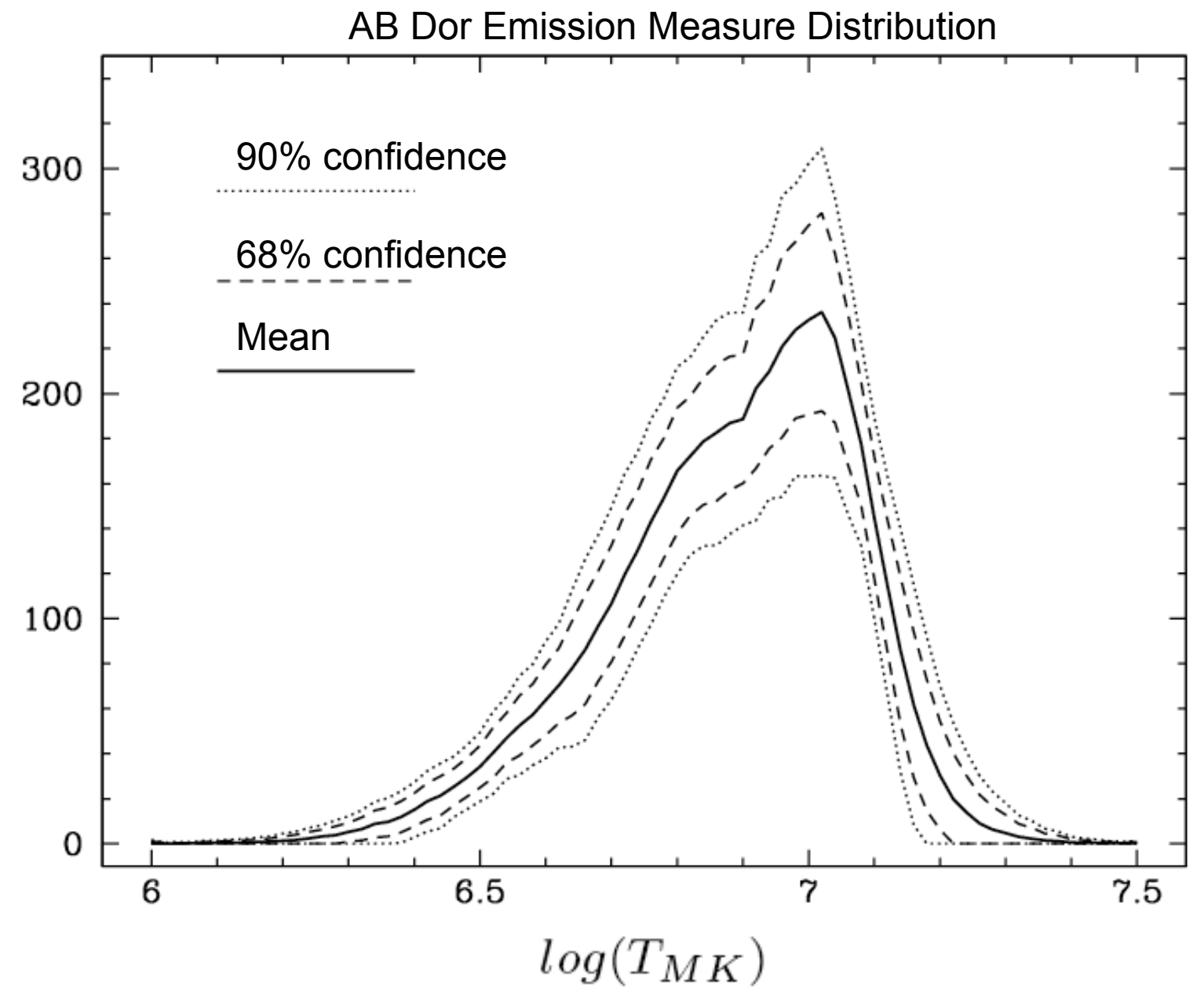


- The Photon Clean Method allows for complicated parameter distributions

- Phase I solution gives best-fit solution from existing models

- Phase Il solution modifies model to quantify amount of departure from physical models

- Bootstrap re-sampling may determine variability of trivial and non-trivial solutions without assumptions about the underlying distribution of the data

- As a test of the PCM's ability to simultaneously model Fe K and Fe L shell line emission, we are using it to model spectra produced by the LLNL EBIT's Maxwellian plasma simulator mode.

\section{Thank You}

